\title{
Sex differences in pain and thermal sensitivity: The role of body size
}

\author{
STEFAN LAUTENBACHER and FRIEDRICH STRIAN \\ Max Planck Institute for Psychiatry, Munich, Germany
}

\begin{abstract}
Sex differences in heat-pain and thermal sensitivity were investigated in 32 women (20 to 60 years of age) and 32 men (17 to 63 years of age) who had no somatosensory impairments. Pain thresholds were measured with stimuli of two different durations (phasic and tonic). Warmth and cold thresholds were assessed as indices of thermal sensitivity. Stimulation was applied to the hand and to the foot by an apparatus containing a Peltier thermode. There were no sex differences in heat-pain thresholds. Women had significantly lower warmth thresholds than men (more pronounced on the foot than on the hand), but similar cold thresholds. Measures of body size (weight, height) correlated much more strongly with thermal than with pain sensitivity, and helped to explain the sex difference in the warmth threshold. A reduction of sex differences to body-measure differences appears likely, but could not be demonstrated unequivocally.
\end{abstract}

There is a widespread belief that women are more responsive to pain than men. According to some authors, this view is supported unequivocally by experimental studies (e.g., Otto \& Dougher, 1985; Velle, 1987). Indeed, women have been found to be more sensitive than men-especially with respect to the pain-tolerance threshold, but also to the pain threshold-in a considerable number of studies (for reviews see Goolkasian, 1985; Rollman \& Harris, 1987; Velle, 1987). But there are also many exceptions to this rule. For instance, with electrocutaneous stimulation, Neri and Agazzani (1984) found no sex differences in the detection threshold, pain threshold, or paintolerance threshold. Their findings were corroborated by Robin, Vinard, Vernet-Maury, and Saumet (1987) for the detection threshold and the pain threshold, but not for the pain-tolerance threshold, where the women had significantly lower values than the men. Furthermore, neither Notermans (1966), with electrocutaneous stimulation, nor Harkins and Chapman (1977), with electrodental stimulation, found any sex differences in the pain threshold. Negative results with respect to sex differences have not been confined to electrical pain-induction techniques, however. In a study using heat-pain stimulation, Clark and Mehl (1971), using a radiation technique, observed similar pain thresholds for women and men, a finding that was also obtained by Kenshalo (1986), who used a contact thermode. Furthermore, in the early studies of Hardy, Wolff, and Goodell (1952), who used radiation heating, no sex differences were found. According to these results, it can at best be said that, if sex differences are to be found at all, women are more responsive to pain than men.

Among the variables that have been suggested as causes of sex differences are anxiety (Robin et al., 1987), sex-role

The authors' mailing address is Max Planck Institute for Psychiatry, Kraepelinstr. 10, D-8000 Munich 40, Germany. expectation (Otto \& Dougher, 1985), and sex hormones (Velle, 1987). These variables have been thought to reflect primary sex characters of perceptual processes. Some authors, however, have proposed that sex differences in pain perception are not attributable to such primary sex characters, but to variables such as body size and skin thickness (Arendt-Nielsen \& Bjerring, 1988; Larkin, Reilly, \& Kittler, 1986). Larkin et al. conducted a thorough analysis of their finding that women had lower detection and "annoyance" thresholds under electrocutaneous stimulation. They found that these sex differences disappeared when the effect of body weight or body-surface area was removed. This suggests that sex differences in somatosensory perception can be explained by variables that influence the results in both sexes in a similar way, and that no special sex variables must be hypothesized.

The major aim of the present study was to look for sex differences in the perception of heat pain and to assess the degree to which any such differences might be explained by differences in body measures. According to Larkin et al. (1986), the variables that explain most of the interindividual variance are body weight and body-surface area. Since the body-surface area is not usually measured but instead is derived from weight and height using an anthropometric formula, and since weight and height are normally highly correlated, we expected even higher correlations between body-surface area and the other two variables. Therefore, we thought it appropriate to use only weight and height as covariates in order to avoid unnecessary multiple testing. We assumed that body-measure effects were due to influences on spatial and temporal summation processes. (Body measures may correlate with the density of the receptive units in the skin and, thereby, with the number of stimulated afferent nerve fibers; and they are related to the nerve conduction time, which influences the temporal process of activation in higher order neurons.) We therefore studied pain perception with heat stimuli of 
different durations (phasic and tonic) so that we could determine the effects of different degrees of temporal summation. Furthermore, to provide for variations in spatial summation, we assessed thermal sensitivity in addition to heat-pain perception, since the size of the stimulated area is more important in the former than in the latter (Chéry-Croze, 1983; Kenshalo, 1984; Kojo \& Pertovaara, 1987). As body-measure influences may differ at different sites of stimulation, we assessed all of the somatosensory measures on the hand and on the foot.

In our data analysis, we first determined the differences between women and men before and after removal of the covariance with weight and height. Then we assessed the degree to which the covariance of the somatosensory and body measures was independent of sex. The more similar the covariance proved to be in the two sexes, the stronger would be the argument that sex differences can be reduced to body-measure differences.

\section{Method}

\section{Subjects}

The subjects were 32 women between 20 and 60 years of age $(M=37.7, S D=11.3)$ and 32 men between 17 and 63 years of age $(M=37.6, S D=13.1)$. The mean weight of the women was $58.0 \mathrm{~kg}(S D=5.7)$ and that of the men, $74.1 \mathrm{~kg}(S D=10.4)$; the corresponding values for height were $166.8 \mathrm{~cm}(S D=6.1)$ and $178.4 \mathrm{~cm}(S D=7.2)$. Subjects with any disorders that could lead to somatosensory impairment were excluded in a neurological investigation.

\section{Apparatus and Procedure}

The stimulator used was a temperature-controlled contact thermode with a stimulation surface of $1.6 \times 3.6 \mathrm{~cm}^{2}$, mounted on an articulated arm. Contact pressure could be adjusted and was held at $0.4 \mathrm{~N} / \mathrm{cm}^{2}$. The apparatus also included a thermode controller with a microcomputer, for managing thermal stimulation, and an IBM personal computer, for controlling the procedures and giving a basic evaluation. Integrated into the response panel were visual signaling devices. Acoustic cues were delivered by the sound generator of the personal computer. The apparatus (PATH Tester MPI 100; for details, see Galfe, Lautenbacher, Hölzl, \& Strian, 1990) had been developed in the Department of Neurology of the Max Planck Institute for Psychiatry in cooperation with Phywe Systeme GmbH (Göttingen).

The two experimenters were male. Each tested half of the subjects. Differing social demands were avoided by having the experimenter read aloud standardized instructions, which stressed that sensory processes were under investigation. No verbal feedback on the results was given during the session; the seating arrangement was such that feedback by facial or other expression was avoided.

The subject sat upright at a table. For measurement on the hand, the subject placed the thenar of his/her hand on the thermode. For measurement on the foot, the thermode was attached to the lateral dorsum pedis with the long edge at a distance of about $1 \mathrm{~cm}$ from the toes.

Determination of the phasic pain threshold. Beginning at a temperature of $40^{\circ} \mathrm{C}$, eight heat stimuli were applied with a rate of temperature change of $0.7^{\circ} \mathrm{C} / \mathrm{sec}$. The subjects were instructed to press a button as soon as they felt pain. Each time they pressed the button, the temperature returned to the base value at a cooling rate of $1.5^{\circ} \mathrm{C} / \mathrm{sec}$. Thus, the nociceptive stimulation was very short.
The start of each trial was announced visually and acoustically, but the stimulus was presented with a pseudorandomized delay of 1 to $3 \mathrm{sec}$. After an intertrial interval of $10 \mathrm{sec}$, the experimenter could start the next trial. With these stimulus intensities and intertrial intervals, the strength of pain perception remains constant over relatively long time periods, indicating that no temporal summation occurs (Lautenbacher, Galfe, Hölzl, \& Strian, 1989). The phasic pain threshold was calculated as the mean of the peak temperatures of the last five stimuli.

Determination of the tonic pain threshold. Starting at a temperature of $40^{\circ} \mathrm{C}$, the subjects adjusted the temperature of the pain threshold using heating and cooling buttons. They indicated their final adjustment by pushing a third button. Then they were stimulated at this temperature for a further $35 \mathrm{sec}$. Characteristic changes in pain perception due to temporal summation occur during intervals of this length (Severin, Lehmann, \& Strian, 1985): If the temperature selected is above the pain threshold, sensation strength normally increases (sensitization), and if the opposite is true, it decreases (adaptation). After this 35-sec period of constant stimulation, the subjects readjusted the temperature to the level of sensation they had experienced when adjusting for the pain threshold the first time (instructions: "The intensity of your sensation may have gotten stronger or weaker. Please adjust the temperature in such a way that you have the same sensation you had after your initial adjustment"). Because the initial adjustment of the pain-threshold temperature usually lasts long enough to activate temporal summation processes, we took the mean of the two adjusted temperatures (initial adjustment and readjustment) as the threshold measure for each trial. The error variance is lower with this measure than it is with measures of a single adjustment. On each trial, the beginning and ending of the two stimulus adjustment periods and of the interval of constant stimulation were signaled visually and acoustically. There were six trials, with intertrial intervals of $15 \mathrm{sec}$. The mean of the last five trials was used as the measure of the tonic pain threshold.

In an earlier study, we had demonstrated that tonic stimuli are perceived as less intense than phasic stimuli of the same temperature in the low heat range (well below the pain threshold) and as more intense in the high heat range and in the pain range (near and above the pain threshold) (Lautenbacher, Möltner, et al., 1989). These differences are attributable to differences in the degree of temporal summation.

Determination of the warmth and cold thresholds. Starting at a temperature of $32^{\circ} \mathrm{C}$, seven warm stimuli and then seven cold stimuli were administered. The rate of the temperature change was again $0.7^{\circ} \mathrm{C} / \mathrm{sec}$. The subjects were told to press a button as soon as they noticed a change in temperature. Thereupon, the temperature returned to the base value $\left(1.5^{\circ} \mathrm{C} / \mathrm{sec}\right)$. The mean differences between the base temperature and the peak temperature in the two sets of seven trials were the measures of the warmth and cold thresholds (the signs of the mean differences were disregarded to enable a better presentation). The intertrial interval lasted $10 \mathrm{sec}$. The stimuli were delayed between 1 and $3 \mathrm{sec}$ (pseudorandomized intervals) after the start of a trial was visually and acoustically signaled.

The thresholds for warmth, cold, and phasic pain were determined in that order, twice a session at each site, always beginning with the hand. The threshold for tonic pain was measured only once at each point of stimulation, after the second assessment of the phasic pain threshold, because this procedure was too time-consuming to have two measurements. The session lasted about $90 \mathrm{~min}$.

\section{Evaluation}

For group comparisons between the sexes, $t$ tests were used. The influence of weight and height on the somatosensory thresholds was evaluated with multiple regression analyses, from which the coefficients of the multiple and partial correlations as well as the residuals 


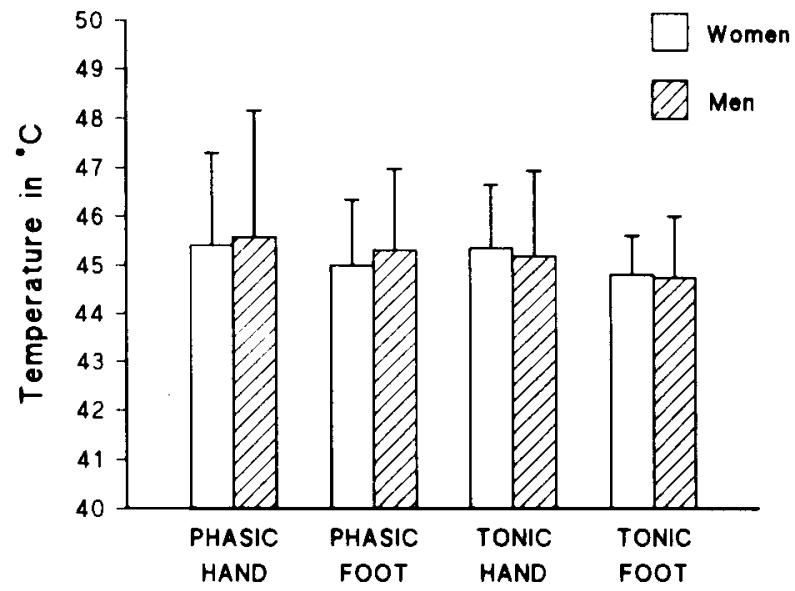

Figure 1. Mean and standard deviation of the phasic and tonic pain thresholds in women and men, measured on the hand and on the foot. Number of measurements: 64 for phasic pain thresholds and 32 for tonic pain thresholds in each group.



Figure 2. Mean and standard deviation of the warmth and cold thresholds, expressed as absolute values of the differences between the threshold temperatures and the base temperature of $32^{\circ} \mathrm{C}$, in women and men, measured on the hand and on the foot. Number of measurements: 64 for each threshold in each group.

(threshold values after the removal of the covariance with weight and height) were taken.

\section{RESULTS}

The somatosensory thresholds of the women and men are shown in Figures 1 and 2. From Figure 1, it is clear that there were no sex differences in either the phasic or the tonic pain thresholds, and that this was true on both the hand and the foot (phasic pain-hand, $p=.657$, foot, $p=.247$; tonic pain-hand, $p=.675$, foot, $p=.811$ ). The findings for the cold threshold were similar in this respect (hand, $p=.121$; foot, $p=.361$; Figure 2). In contrast, the women had lower warmth thresholds than the men, the difference being significant on the hand $(p=.042)$ and very highly significant on the foot $(p<.001$; Figure 2).

Table 1 shows that the body measures (weight and height) did not covary markedly with the pain thresholds (phasic and tonic). However, highly to very highly significant multiple correlations were found for the thermal thresholds. The partial correlations suggest a positive relationship between thermal thresholds and weight. Height seemed to influence only the cold threshold, here negatively.

After removal of the covariance with the body measures, sex differences were assessed again with the residuals of the somatosensory thresholds resulting from the multiple regression analysis. Table 2 presents the sex differences (men minus women) before and after this correction. With the residuals, no significant sex differences were found (phasic pain-hand, $p=.812$, foot, $p=.493$; tonic painhand, $p=.705$, foot, $p=.943$; warmth-hand, $p=.981$, foot, $p=.166$; cold-hand, $p=.600$; foot, $p=.372$ ). This means that the only significant sex diferences-those observed for the warmth thresholds-disappeared after the body-measure correction.

To prove that the influence of body measures on the somatosensory thresholds is independent of sex, we

Table 1

Results of the Multiple Regression Analyses with Body Weight and Body Height as Predictors and the Sensory Thresholds as Criteria

\begin{tabular}{lcrr}
\hline & $\begin{array}{c}\text { Multiple } \\
\text { Correlation }\end{array}$ & \multicolumn{2}{c}{ Partial Correlation } \\
\cline { 3 - 4 } & & & Weight \\
\hline $\begin{array}{l}\text { Phasic pain } \\
\text { Hand }\end{array}$ & 0.037 & -0.014 & 0.035 \\
$\begin{array}{l}\text { Foot } \\
\text { Tonic pain }\end{array}$ & 0.169 & 0.168 & -0.130 \\
$\quad$ Hand & & & \\
Foot & 0.141 & 0.122 & -0.135 \\
Warmth & 0.208 & 0.172 & -0.203 \\
$\quad$ Hand & & & \\
Foot & $0.304 \dagger$ & $0.278 \dagger$ & -0.096 \\
Cold & $0.456 \ddagger$ & $0.413 \ddagger$ & -0.133 \\
$\quad$ Hand & & & \\
Foot & $0.375 \ddagger$ & $0.365 \ddagger$ & $-0.192 *$ \\
\hline
\end{tabular}

Note-Coefficients $(r)$ for the multiple and partial correlations. Number of measurements: 128 for the phasic pain, warmth, and cold thresholds, 64 for the tonic pain threshold. ${ }^{*} p \leq .05$. $\ddagger p \leq .01 . \ddagger p \leq .001$

Table 2

Differences in the Mean Sensory Thresholds of Men and Women (Men minus Women) Before and After Body-Measure Correction, in Degrees Centigrade

\begin{tabular}{lrrrrr}
\hline & \multicolumn{2}{c}{ Uncorrected } & & \multicolumn{2}{c}{ Corrected } \\
\cline { 2 - 3 } \cline { 6 - 6 } & Hand & Foot & & Hand & \multicolumn{1}{c}{ Foot } \\
\hline Phasic pain & 0.18 & 0.31 & & 0.10 & 0.18 \\
Tonic pain & -0.16 & -0.06 & & -0.14 & -0.02 \\
Warmth & 0.32 & 2.15 & & $<0.01$ & 0.61 \\
Cold & 0.10 & 0.21 & & -0.03 & -0.19 \\
\hline
\end{tabular}

Note-For body-measure correction, the residuals of the thresholds in the multiple regression analyses with body weight and body height as predictors were used. Number of measurements in each group: 64 for the phasic pain, warmth, and cold thresholds, 32 for the tonic pain threshold. 
Table 3

Results of the Multiple Regression Analyses with Body Weight and Body Height as Predictors and the Sensory Thresholds as Criteria, Computed Separately for Women (W) and Men (M)

\begin{tabular}{|c|c|c|c|c|}
\hline & & \multirow{2}{*}{$\begin{array}{c}\text { Multiple } \\
\text { Correlation }\end{array}$} & \multicolumn{2}{|c|}{ Partial Correlation } \\
\hline & & & Weight & Height \\
\hline \multicolumn{5}{|c|}{ Phasic pain } \\
\hline \multirow[t]{2}{*}{ Hand } & W & 0.059 & 0.052 & -0.004 \\
\hline & $\mathbf{M}$ & 0.055 & -0.055 & 0.019 \\
\hline \multirow[t]{2}{*}{ Foot } & $\mathbf{W}$ & 0.301 & $0.271^{*}$ & $-0.266^{*}$ \\
\hline & $\mathbf{M}$ & 0.120 & 0.054 & -0.119 \\
\hline \multicolumn{5}{|c|}{ Tonic pain } \\
\hline \multirow[t]{2}{*}{ Hand } & W & 0.291 & 0.285 & -0.113 \\
\hline & $\mathbf{M}$ & 0.147 & 0.093 & -0.140 \\
\hline \multirow[t]{2}{*}{ Foot } & W & 0.378 & 0.230 & $-0.378^{*}$ \\
\hline & $\mathbf{M}$ & 0.146 & 0.142 & -0.080 \\
\hline \multicolumn{5}{|l|}{ Warmth } \\
\hline \multirow[t]{2}{*}{ Hand } & W & 0.131 & 0.110 & -0.001 \\
\hline & $\mathbf{M}$ & $0.335^{*}$ & $0.333 \dagger$ & -0.155 \\
\hline \multirow[t]{2}{*}{ Foot } & W & $0.493 \ddagger$ & $0.492 \ddagger$ & $-0.335 \dagger$ \\
\hline & $\mathbf{M}$ & 0.266 & $0.255^{*}$ & -0.165 \\
\hline \multicolumn{5}{|l|}{ Cold } \\
\hline \multirow[t]{2}{*}{ Hand } & W & $0.345^{*}$ & $0.318^{*}$ & -0.061 \\
\hline & $\mathbf{M}$ & $0.446 \dagger$ & $0.428 \ddagger$ & $-0.296^{*}$ \\
\hline \multirow[t]{2}{*}{ Foot } & W & $0.402 \dagger$ & $0.399 \dagger$ & $-0.277^{*}$ \\
\hline & $\mathbf{M}$ & $0.450 \ddagger$ & $0.450 \ddagger$ & -0.166 \\
\hline
\end{tabular}

Note-Coefficients $(r)$ of the multiple and partial correlations. Number of measurements: 64 for the phasic pain, warmth, and cold thresholds, 32 for the tonic pain threshold. $* p \leq .05$. $\quad t p \leq .01$. $\ddagger p \leq .001$.

repeated the regression analyses for each sex separately. The results are given in Table 3. As far as can be seen from a regression analysis on a sample of this size, the influence of body measures is similar. It is true that significant covariations with the pain thresholds were found only in the group of women. For the thermal thresholds, however, the findings, with some exceptions, were more homogeneous. Both sexes had significant covariations of weight with the warmth threshold on the foot and with the cold thresholds on the hand and on the foot; the linear combination of weight and height had significant correlations with the cold threshold on the hand and on the foot. This also means that in the case of the warmth threshold on the foot, where the only marked sex difference before body-measure correction was found, weight seemed to influence the threshold in both sexes.

\section{DISCUSSION}

Our main finding relating to pain sensitivity is that there were no sex differences in pain threshold at either site (hand, foot), with either kind of stimulation (phasic, tonic). Thus, our study supports the view that there are not necessarily any sex differences in pain perception and that apparent differences seem to depend on additional variables (Clark \& Mehl, 1971; Harkins \& Chapman, 1977; Kenshalo, 1986). Interestingly, with the kind of heat-pain measurement we used, body measures appeared to have little influence on the results - with the exception of some rather inconsistent findings in the women. In contrast, Larkin et al. (1986) found significant covariation between body size and "annoyance" threshold, which helped to explain the observed sex differences in the threshold values. One conclusion might be that painmeasurement techniques with little or no influence of body measures on the results produce no sex differences, whereas other techniques produce sex differences via body-measure differences. So far, however, this hypothesis has an empirical basis for the pain threshold only and not for other parameters of pain perception.

We found sex differences in thermal sensitivity for the warmth threshold, but not for the cold threshold, and this difference was more pronounced on the foot than on the hand. This finding is compatible with the findings of other authors: In a variety of somatosensory tests (touch, vibration, heat pain, warmth, and cold, measured on the hand and on the foot), Kenshalo (1986) found a significant sex difference only for the warmth threshold on the foot. Other authors, too (Dyck, Karnes, \& O'Brien, 1987; Gray, Stevens, \& Marks, 1982), were unable to find any sex differences in cold thresholds measured at various anatomic sites. Only our finding of a weak, but nevertheless significant, sex difference in the warmth threshold on the hand was not obtained in the other studies (Gray et al., 1982; Kenshalo, 1986). We speculate that the different results on warmth and cold sensitivity in this respect may depend on differences in their respective spatial summation capacities. The area at which spatial summation reaches its ceiling appears to be somewhat smaller for cold than for warmth sensitivity and to fall into the range of areas stimulated by the usual thermal contact devices (Greenspan \& Kenshalo, 1985; Kenshalo, 1984). Therefore, it is more likely that spatial summation is already saturated for cold than for warmth sensitivity, making the former presumably less susceptible to body-size influences and, thereby, to sex differences.

In our view, it is unlikely that the higher warmth thresholds in men reflect sex differences in response criterion. First of all, in the other studies, the findings similar to ours were obtained with forced-choice procedures (Dyck et al., 1987; Gray et al., 1982; Kenshalo, 1986), which are said to be resistant to response-bias influences. Secondly, in a recent study, it was shown that results obtained with our threshold procedure and with a forcedchoice procedure do not differ very much (Claus, Hilz, \& Neundörfer, 1990).

For the thermal thresholds-in contrast to the pain thresholds-a strong covariation with the body measures could be demonstrated at both anatomic sites in the combined sample. Weight appeared to be related to the warmth and the cold thresholds in a positive fashion; skin thickness and density of the receptive units in the skin might be the intervening variables (see the introduction above). The negative relation between height and cold threshold seems surprising, but it was derived from a linear combination of weight and height used for predicting the threshold, and both variables were strongly correlated $(r=.68)$. Therefore, height is only a necessary correction factor in the overall positive relation between body size and cold threshold. Our assumption that we would 
gain no further information by using the body-surface area computed from weight and height seems to be supported by correlations of $r=.96$ for area and weight and $r=.86$ for area and height.

After removal of the covariance with the body measures, the only significant sex difference-that for the warmth threshold-disappeared. This result is similar to that obtained by Larkin et al. (1986) with electrocutaneous stimulation, where the sex differences in the detection and the "annoyance" threshold could be removed in a similar way. Interestingly, Sosenko, Kato, Soto, and Ayyar (1989), who also used a thermal contact stimulation, but one with a stimulation area on the hallux that varied with the size of this part of the body, found neither body-size influences (body-mass index, height) nor sex differences for thermal sensitivity. These findings point clearly to an explanation of sex differences by body-measure differences. However, in a short communication, Rollman, Hapidou, and Jarmain (1990) reported a failure of their attempt to explain sex differences by body-size differences, although they measured the electrocutaneous detection threshold just as Larkin et al. (1986) did. In addition, our findings allow us to state only that the body-measure influences on somatosensory thresholds are similar in the two sexes, and this is another warning against trying to oversimplify this issue. We think that sex independence of body-measure influences is a prerequisite for a simple reduction of sex differences to body-size differences. Nevertheless, our study, together with that of Larkin et al., provides reason enough to use body measures in the explanation of sex differences first and more complex explanations only if the remaining unexplained sex variance justifies such an approach.

Taken together, the findings of our study are a further refutation of the belief that women are generally more responsive to pain than men, at least in experimental pain studies. Rather, the type, intensity, and context of painful stimulation and certain characteristics of the subject other than sex also seem to be important determinants of the results. The significant sex differences in warmth (but not in cold) thresholds that we found fit well with earlier findings on sex differences in thermal sensitivity. Our attempt to reduce these differences to body-measure differences was successful and corroborates the findings of Larkin et al. (1986). However, a complete absence of any sex dependence of body-measure influences on somatosensory thresholds, a prerequisite for a purely reductionistic view, could not be demonstrated unequivocally.

\section{REFERENCES}

Arendt-Nielsen, L., \& BJerring, P. (1988). Sensory and pain threshold characteristics to laser stimuli. Journal of Neurology, Neurosurgery, \& Psychiarry, 51, 35-42.

ChÉry-Croze, S. (1983). Painful sensation induced by a thermal cutaneous stimulus. Pain, 17, 109-137.

Clark, W. C., \& Mehl, L. (1971). Thermal pain: A sensory decision analysis of the effect of age and sex on $\mathrm{d}^{\prime}$, various response criteria, and 50\% pain threshold. Journal of Abnormal Psychology, 78, 202-212.
Claus, D., Hilz, M. J., \& Neundörfer, B. (1990). Thermal discrimination thresholds: A comparison of different methods. Acta Neurologica Scandinavica, 81, 533-540.

Dyck, P. J., KARNes, J., \& O'BRIEN, P. C. (1987). Detection thresholds of cutaneous sensation. In P. J. Dyck, P. K. Thomas, A. K. Asbury, A. I. Winegrad, \& D. Porte (Eds.), Diabetic neuropathy (pp. 107121). Philadelphia: Saunders.

Galfe, G., Lautenbacher, S., Hölzl, R., \& Strian, F. (1990). Diagnosis of small fibre neuropathy: Computer-assisted methods of combined pain and thermal sensitivity determination. Hospimedica, 8(7). 38-48.

Goolkasian, P. (1985). Phase and sex effects in pain perception: A critical review. Psychology of Women Quarterly, 9, 15-28.

Gray, L., Stevens, J. C., \& Marks, L. E. (1982). Thermal stimulus thresholds: Sources of variability. Physiology \& Behavior, 29, 355-360.

Greenspan, J. D., \& Kenshalo, D. R., Sr. (1985). The primate as a model for the human temperature-sensing system: 2 . Area of skin receiving thermal stimulation (spatial summation). Somatosensory Research, 2, 315-324.

Hardy, J. D., WolfF, H. G., a Goodell, H. (1952). Pain sensations and reactions. Battimore: Williams \& Wilkins.

Harkins, S. W., Chapman, C. R. (1977). Age and sex differences in pain perception. In D. J. Anderson \& B. Matthews (Eds.), Pain in the trigeminal region (pp. 435-441). Amsterdam: Elsevier/NorthHolland Biomedical Press

KenShalo, D. R., SR. (1984). Cutaneous temperature sensitivity. In W. W. Dawson \& J. M. Enoch (Eds.), Foundations of sensory science (pp. 419-464). Berlin: Springer-Verlag

Kenshalo, D. R., SR. (1986). Somesthetic sensitjity in young and elderly humans. Journal of Gerontology, 41, 732-742.

Kojo, I., \& Pertovana, A. (1987). The effects of stimulus area and adaptation temperature on warm and heat pain thresholds in man. International Journal of Neuroscience, 32, 875-880.

Larkin, W. D., Reilly, J. P., \& Kittler, L. B. (1986). Individual differences in sensitivity to transient electrocutaneous stimulation. IEEE Transactions on Biomedical Engineering, 33, 495-504.

Lautenbacher, S., Galfe, G., Hölzl, R., Strian, F. (1989). Threshold tracking for assessment of long-term adaptation and sensitization in pain perception. Perceptual \& Motor Skills, 69, 579-589.

Lautenbacher, S., Möltner, A., Lehmann, W. P., Galfe, G., HölzL, R., \& Strian, F. (1989). SDT analysis of experimental thermal pain, with "signal" and "no-signal" being determined psychophysically. Perceptual \& Motor Skills, 68, 1019-1030.

Neri, M., \& Agazzani, E. (1984). Aging and right-left asymmetry in experimental pain measurement. Pain, 19, 43-48.

Notermans, S. L. H. (1966). Measurement of the pain threshold determined by electrical stimulation and its clinical application: Part 1 . Method and factors possibly influencing the pain threshold. Neurology, 16, 1071-1086.

OTto, M. W., DOUGHER, M. J. (1985). Sex differences and personality factors in responsivity to pain. Perceptual \& Motor Skills, 61, 383-390.

Robin, O., Vinard, H., Vernet-MaURy, E., \& SAumet, J.-L. (1987) Influence of sex and anxiety on pain threshold and tolerance. Functional Neurology, 2, 173-179.

Rollman, G. B., Hapidou, E. G., \& Jarmain, S. H. (1990). Gender differences in pain responsiveness: Contributing factors. Pain (Suppl. 5), 314.

Rollman, G. B., \& Harris, G. (1987). The detectability, discriminability, and perceived magnitude of painful electrical shock. Perception \& Psychophysics, 42, 257-268.

Severin, F., Lehmann, W. P., Strian, F. (1985). Subjective sensitization to tonic heat as an indicator of thermal pain. Pain, 21 , 369-378.

Sosenxo, J. M., Kato, M., Soto, R., \& Ayyar, D. R. (1989). Determinants of quantitative sensory testing in nonneuropathic individuals. Electromyography \& Clinical Neurophysiology, 29, 459-463.

VELLE, W. (1987). Sex differences in sensory functions. Perspectives in Biology \& Medicine, 30, 490-522.

(Manuscript received October 9, 1990; revision accepted for publication March 8, 1991.) 